\title{
Logarithmic cohomology of the complement of a plane curve
}

Francisco J. Calderón Moreno ${ }^{1, *}$, David Mond, Luis Narváez Macarro ${ }^{1}$ and Francisco J. Castro Jiménez ${ }^{1}$

\begin{abstract}
Let $D, x$ be a plane curve germ. We prove that the complex $\Omega^{\bullet}(\log D)_{x}$ computes the cohomology of the complement of $D, x$ only if $D$ is quasihomogeneous. This is a partial converse to a theorem of [5], which asserts that this complex does compute the cohomology of the complement, whenever $D$ is a locally weighted homogeneous free divisor (and so in particular when $D$ is a quasihomogeneous plane curve germ). We also give an example of a free divisor $D \subset \mathbb{C}^{3}$ which is not locally weighted homogeneous, but for which this (second) assertion continues to hold.
\end{abstract}

Mathematics Subject Classification (2000). Primary 32S20; Secondary 32S40, 14F40.

Keywords. Free divisor, logarithmic de Rham complex, plane curve, local quasi-homogeneity.

\section{Introduction}

In [5] the last three authors showed that if $D$ is a locally quasi-homogeneous free divisor in the complex manifold $X$ then locally the complex $\Omega^{\bullet}(\log D)$ of holomorphic differential forms with logarithmic poles along $D$ calculates the cohomology of the complement of $D$ in $X$. More precisely, the following two equivalent statements hold:

Theorem 1.1. With $D$ as above,

1. If $V \subset X$ is a Stein open set then the de Rham map (integration of forms over cycles) gives rise to an isomorphism

$$
h^{k}\left(\Gamma\left(V, \Omega^{\bullet}(\log D)\right)\right) \stackrel{\sim}{\rightarrow} H^{k}(V \backslash D ; \mathbb{C}) .
$$

2. Denoting by $U$ the complement of $D$ in $X$ and by $j: U \hookrightarrow X$ the inclusion, the de Rham morphism gives rise to an isomorphism

$$
\Omega^{\bullet}(\log D) \stackrel{\sim}{\rightarrow} \mathbf{R} j_{*}\left(\mathbb{C}_{U}\right) .
$$

\footnotetext{
*Supported by MEC of Spain and EPSRC of United Kingdom.

${ }^{1}$ Partially supported by PB97-0723.
} 
By analogy with Grothendieck's Comparison Theorem [8], in which the complex $\Omega^{\bullet}(\log D)$ is replaced in these two statements by $\Omega^{\bullet}(* D)$, but which holds for an arbitrary divisor, we summarise this with a slogan: if $D \hookrightarrow X$ is a locally quasihomogeneous free divisor then the logarithmic comparison theorem holds.

The definition of local quasi-homogeneity, (called strong quasi-homogeneity in $[5])$, is as follows:

\section{Definition 1.2.}

1. The polynomial $h\left(z_{1}, \cdots, z_{n}\right)=\sum a_{i_{1}, \cdots, i_{n}} z_{1}^{i_{1}} \cdots z_{n}^{i_{n}} \in \mathcal{O}_{\mathbb{C}^{n}}$ is weighted homogeneous if there exist positive integer weights $w_{1}, \cdots, w_{n}$ such that $h\left(z_{1}^{w_{1}}, \cdots, z_{n}^{w_{n}}\right)$ is homogeneous.

2. The divisor $D \subset X$ is locally quasi-homogeneous if for all $x \in D$ there are local coordinates on $X$, centered at $x$, with respect to which $D$ has a weighted homogeneous defining equation.

Every plane curve is a free divisor, since the module of logarithmic vector fields $\operatorname{Der}(\log D)$ is reflexive and thus has depth at least 2. In [4, Cor. 4.2.2] the first author showed that if $D$ is a plane curve then the logarithmic de Rham complex $\Omega^{\bullet}(\log D)$ is perverse, a necessary condition for the logarithmic comparison theorem.

In [6] the logarithmic comparison theorem has been tested for the following non locally quasi-homogeneous plane curve (cf. [9]): $D=\left\{f=x_{1}^{4}+x_{2}^{5}+x_{2}^{4} x_{1}=\right.$ $0\} \subset X=\mathbb{C}^{2}$. A basis for $\mathcal{D} \operatorname{er}(\log D)$ is given by:

$$
\begin{aligned}
& \delta_{1}=\left(16 x_{1}^{2}+20 x_{1} x_{2}\right) \frac{\partial}{\partial x_{1}}+\left(12 x_{1} x_{2}+16 x_{2}^{2}\right) \frac{\partial}{\partial x_{2}} \\
& \delta_{2}=\left(16 x_{1} x_{2}^{2}+4 x_{2}^{3}-125 x_{1} x_{2}\right) \frac{\partial}{\partial x_{1}}+\left(12 x_{2}^{3}-4 x_{1}^{2}+5 x_{1} x_{2}-100 x_{2}^{2}\right) \frac{\partial}{\partial x_{2}} .
\end{aligned}
$$

Let $\mathcal{D}_{X}$ be the sheaf of linear differential operators with holomorphic coefficients on $X$ and $I$ the left $\mathcal{D}_{X}$-ideal generated by $\delta_{1}, \delta_{2}$. By [4, Th. 4.2.1], we have a (canonical) isomorphism (in the derived category)

$$
\Omega^{\bullet}(\log D) \simeq \mathbf{R} \operatorname{Hom}_{\mathcal{D}_{X}}\left(\mathcal{D}_{X} / I, \mathcal{O}_{X}\right)
$$

and so we can compute the characteristic cycle $C C\left(\Omega^{\bullet}(\log D)\right)$ as the cycle $Z$ in $T^{*} X$ determined by the ideal $J=\sigma(I)$ generated by the principal symbols of elements in $I$. The symbols $\sigma_{1}=\sigma\left(\delta_{1}\right), \sigma_{2}=\sigma\left(\delta_{2}\right)$ form a regular sequence in $\mathcal{O}_{T^{*} X}$ and so, by [4, Prop. 4.1.2], the ideal $J$ is generated by $\sigma_{1}, \sigma_{2}$. An easy computation shows that the multiplicity of the conormal at 0 in $Z$ is 4 . On the other hand, the multiplicity of the conormal at 0 in $C C\left(\mathbf{R} j_{*}\left(\mathbb{C}_{U}\right)\right)$ is equal to mult $_{0}(D)-1=3$ (cf. [3]), and so the logarithmic comparison theorem does not hold for $D$.

For the family of non locally quasi-homogeneous plane curves (cf. [9])

$$
x_{1}^{q}+x_{2}^{p}+x_{2}^{p-1} x_{1}=0, \quad p \geq q+1 \geq 5,
$$


the multiplicities of the conormal at 0 in $C C\left(\Omega^{\bullet}(\log D)\right)$ and in $C C\left(\mathbf{R} j_{*}\left(\mathbb{C}_{U}\right)\right)$ are $2(q-2)$ and $q-1$ respectively, and so these curves also do not satisfy the logarithmic comparison theorem.

A natural question is therefore whether or not the logarithmic comparison theorem holds for a given free divisor.

The purpose of this paper is to prove a partial converse to Theorem 1.1. We prove:

Theorem 1.3. Let $D$ be a reduced plane curve. If the logarithmic comparison theorem holds for $D$, then $D$ is locally quasi-homogeneous.

Our proof shows that if $h$ is a local equation of $D$, and the logarithmic comparison theorem holds, then there is a vector field germ $\chi$ such that $\chi \cdot h=h$. As a reduced curve has isolated singularities, we can then apply the theorem of K. Saito [10]: if $h \in \mathcal{O}_{\mathbf{C}^{n}, 0}$ has isolated singularity and $h$ belongs to its Jacobian ideal $J_{h}$ then in suitable coordinates $h$ is weighted homogeneous.

We conjecture that in higher dimensions the following version of our Theorem 1.3 holds:

Conjecture 1.4. If $D \hookrightarrow X$ is a free divisor and if the logarithmic comparison theorem holds, then for all $x \in D$ there is a local equation $h$ for $D$ around $x$, and a germ of vector field $\chi$ vanishing at $x$ such that $\chi \cdot h=h$.

A singular free divisor of dimension greater than 1 has non-isolated singularities, so even if this conjecture is true, Saito's theorem cannot be used to deduce local quasi-homogeneity. Indeed, it is not true in higher dimensions that if the logarithmic comparison theorem holds for a free divisor $D$ then $D$ is necessarily locally quasi-homogeneous. This is shown by an example in Section 4 below: the logarithmic comparison theorem holds for the free divisor

$$
D=\{(x, y, z): x y(x+y)(z x+y)=0\}
$$

(the total space of a family of four lines in the plane with varying cross-ratio, cf. [4]), in the neighbourhood of $(0,0, \lambda)$, with $\lambda \in \mathbb{C} \backslash\{0,1\}$; however it is well known that this divisor is not locally quasi-homogeneous. On the other hand, it does satisfy Conjecture 1.4.

Adrian Langer has indicated to us that he has subsequently found a shorter proof of Theorem 1.3, using globalisation and a comparison of Chern classes ${ }^{1}$.

\footnotetext{
${ }^{1}$ Added on November 2001.
} 


\section{Preliminary results}

In this section we recall the spectral sequence argument used in [5] to compare the cohomology of the logarithmic complex $\Omega^{\bullet}(\log D)$ with the cohomology of $X \backslash D$. Except for referring to "local" rather than "strong" quasi-homogeneity, we will use the same notation as [5].

Without loss of generality we assume $X=\mathbb{C}^{n}$ with coordinates $z_{i}$ and $x_{0}=0$. Let $V$ be a Stein neighbourhood (sufficiently small) of 0 , let $\mathcal{U}$ be the open cover of $V \backslash\{0\}$ consisting of the sets $U_{i}=V \cap\left\{z_{i} \neq 0\right\}$, and let $\mathcal{U}^{\prime}$ be the open cover of $V \backslash D$ consisting of the open sets $U_{i}^{\prime}=(V \backslash D) \cap\left\{z_{i} \neq 0\right\}=U_{i} \backslash D$.

We consider the two double complexes

$$
K^{p, q}=\check{C}^{q}\left(\mathcal{U}, \Omega^{p}(\log D)\right)
$$

and

$$
\tilde{K}^{p, q}=\check{C}^{q}\left(\mathcal{U}^{\prime}, \Omega^{p}\right),
$$

equipped with the exterior derivative $d$ (the horizontal differential) and the Čech differential $\delta$ (the vertical differential). There is an obvious restriction morphism $\rho_{p, q}: K^{p, q} \rightarrow \tilde{K}^{p, q}$ which commutes with both differentials, and thus gives rise to morphisms of the two spectral sequences arising from each double complex. These spectral sequences have $E_{1}$ terms

$$
\begin{aligned}
& { }^{\prime} E_{1}^{p, q}=\check{H}^{q}\left(\mathcal{U}, \Omega^{p}(\log D)\right) \\
& { }^{\prime} \tilde{E}_{1}^{p, q}=\check{H}^{q}\left(\mathcal{U}^{\prime}, \Omega^{p}\right) \\
& { }^{\prime} E_{1}^{p, q}=\oplus_{1 \leq i_{1}<\cdots<i_{q+1} \leq n} h^{p}\left(\Gamma\left(\bigcap_{j} U_{i_{j}}, \Omega^{\bullet}(\log D)\right)\right) \\
& { }^{\prime} \tilde{E}_{1}^{p, q}=\oplus_{1 \leq i_{1}<\cdots<i_{q+1} \leq n} h^{p}\left(\Gamma\left(\bigcap_{j} U_{i_{j}}^{\prime}, \Omega^{\bullet}\right)\right) .
\end{aligned}
$$

As both $\mathcal{U}$ and $\mathcal{U}^{\prime}$ are Stein covers,

$$
\check{H}^{q}\left(\mathcal{U}, \Omega^{p}(\log D)\right)=\check{H}^{q}\left(V \backslash\{0\}, \Omega^{p}(\log D)\right)
$$

and

$$
\left.\left.\check{H}^{q}\left(\mathcal{U}^{\prime}, \Omega^{p}\right)\right)=\check{H}^{q}\left(V \backslash D, \Omega^{p}\right)\right) .
$$

As $V \backslash D$ is Stein, $\check{H}^{q}\left(V \backslash D, \Omega^{p}\right)=0$ if $q>0$. It follows that

$$
\text { " } \tilde{E}_{2}^{p, q}=\left\{\begin{array}{ll}
H^{p}(V \backslash D ; \mathbb{C}) & \text { if } q=0 \\
0 & \text { if } q \neq 0
\end{array},\right.
$$

and in particular the spectral sequence " $\tilde{E}$ converges to the cohomology of $V \backslash D$.

Now assume that outside $0, D$ is locally quasi-homogeneous, so that by 1.1 $\mathbf{R} j_{*}\left(\mathbb{C}_{U}\right) \simeq \Omega^{\bullet}(\log D)$, again outside 0 . As $\mathcal{U}$ and $\mathcal{U}^{\prime}$ are Stein covers, by 1.1 the quotient of the restriction $\rho_{p, q}$ defines an isomorphism ${ }^{\prime} \rho_{p, q}:{ }^{\prime} E_{1}^{p, q} \rightarrow{ }^{\prime} \tilde{E}_{1}^{p, q}$ for all $p, q$. This isomorphism persists to give an isomorphism of the cohomology of the 
total complexes $K^{\text {tot }}$ and $\tilde{K}^{\text {tot }}$ as calculated by the spectral sequences. It follows that the spectral sequence " $E$, like " $\tilde{E}$, also converges to the cohomology of $V \backslash D$ :

$$
H^{k}(V \backslash D ; \mathbb{C}) \simeq \oplus_{p+q=k}{ }^{\prime \prime} E_{\infty}^{p, q} .
$$

As $D$ is a free divisor, $\check{H}^{q}\left(V \backslash\{0\}, \Omega^{p}(\log D)\right)=0$ for $q \neq 0, n-1$, so ${ }^{\prime \prime} E_{1}$ has only two non-null rows; writing for the moment $\Omega^{p}(D)$ and $V^{*}$ in place of $\Omega^{p}(\log D)$ and $V \backslash\{0\},{ }^{\prime \prime} E_{1}$ thus looks like

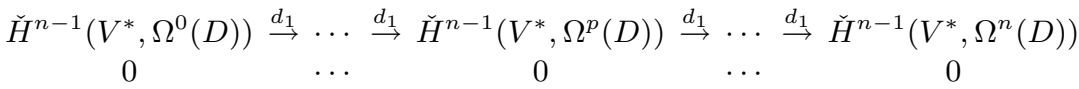

$$
\begin{aligned}
& \begin{array}{ccccc}
\vdots & \vdots & \vdots & \vdots & \vdots \\
0 & \ldots & 0 & \ldots & 0
\end{array} \\
& \Gamma\left(V, \Omega^{0}(D)\right) \quad \stackrel{d_{1}}{\rightarrow} \cdots \stackrel{d_{1}}{\rightarrow} \Gamma\left(V, \Omega^{p}(\log D)\right) \stackrel{d_{1}}{\rightarrow} \cdots \stackrel{d_{1}}{\rightarrow} \Gamma\left(V, \Omega^{n}(\log D)\right) .
\end{aligned}
$$

(Note that as $n \geq 2$ and as the $\Omega^{p}(\log D)$ are free modules, we have $\Gamma\left(V^{*}, \Omega^{p}(D)\right)=$ $\Gamma\left(V, \Omega^{p}(D)\right)$.)

As this spectral sequence converges to the cohomology of $V \backslash D$, we have

$$
\begin{gathered}
H^{n-1}(V \backslash D ; \mathbb{C}) \simeq E_{\infty}^{0, n-1} \oplus \cdots \oplus E_{\infty}^{n-1,0}=E_{n+2}^{0, n-1} \oplus h^{n-1}\left(\Gamma\left(V, \Omega^{\bullet}(\log D)\right)\right) \\
H^{n}(V \backslash D ; \mathbb{C})=E_{\infty}^{0, n} \oplus \cdots \oplus E_{\infty}^{0, n}=E_{n+2}^{1, n-1} \oplus \frac{h^{n}\left(\Gamma\left(V, \Omega^{\bullet}(\log D)\right)\right)}{d_{n+1}\left(E_{n+2}^{0, n-1}\right)},
\end{gathered}
$$

where

$$
E_{n+2}^{0, n-1}=\operatorname{Ker} d_{1}: \check{H}^{n-1}\left(V^{*}, \Omega^{0}(D)\right) \rightarrow \check{H}^{n-1}\left(V^{*}, \Omega^{1}(D)\right) .
$$

In [5], the main theorem was proved by showing that if $D$ is locally quasi-homogeneous then the complex

$$
\left(\check{H}^{n-1}\left(V \backslash\{0\}, \Omega^{\bullet}(\log D)\right), d_{1}\right)
$$

is exact.

\section{Proof of the Theorem}

We continue with the discussion of the last paragraph. If the natural morphism $\Omega^{\bullet}(\log D) \rightarrow \mathbf{R} j_{*}\left(\mathbb{C}_{U}\right)$ is a quasi-isomorphism (i.e. if the logarithmic comparison theorem holds for $D)$ then by the formulae of the last section, $d_{1}: \check{H}^{n-1}(V \backslash$ $\left.\{0\}, \Omega^{0}(\log D)\right) \rightarrow \check{H}^{n-1}\left(V \backslash\{0\}, \Omega^{1}(\log D)\right)$ is injective.

Let $\left\{\omega_{1}, \cdots, \omega_{n}\right\}$ be a free basis of $\Omega^{1}(\log D)$ as $\mathcal{O}_{V}$-module, and let $\delta_{1}, \cdots, \delta_{n}$ be the dual basis of $\mathcal{D} \operatorname{er}(\log D)$. Then $\check{H}^{n-1}\left(V \backslash\{0\}, \Omega^{0}(\log D)\right)=\check{H}^{n-1}(V \backslash$ $\left.\{0\}, \mathcal{O}_{\mathbb{C}^{n}}\right)$ and $\check{H}^{n-1}\left(V \backslash\{0\}, \Omega^{1}(\log D)\right) \simeq \oplus_{1}^{n} \check{H}^{n-1}\left(V \backslash\{0\}, \mathcal{O}_{\mathbb{C}^{n}}\right)$. The morphism $d_{1}: \check{H}^{n-1}\left(V \backslash\{0\}, \Omega^{0}(\log D)\right) \rightarrow \check{H}^{n-1}\left(V \backslash\{0\}, \Omega^{1}(\log D)\right)$ now becomes

$$
\begin{aligned}
\check{H}^{n-1}\left(V \backslash\{0\}, \mathcal{O}_{\mathbb{C}^{n}}\right) & \stackrel{d_{1}}{\rightarrow} \check{H}^{n-1}\left(V \backslash\{0\}, \mathcal{O}_{\mathbb{C}^{n}}\right)^{n} \\
{[g] } & \mapsto\left(\left[\delta_{1} \cdot g\right], \cdots,\left[\delta_{n} \cdot g\right]\right) .
\end{aligned}
$$


where $g \in \Gamma\left(V \backslash \cup_{i}\left\{z_{i}=0\right\}, \mathcal{O}_{\mathbb{C}^{n}}\right)=\Gamma\left(\mathbb{C}^{n} \backslash \cup_{i}\left\{z_{i}=0\right\}, \mathcal{O}_{\mathbb{C}^{n}}\right)$ represents the class $[g]$ in $\tilde{H}^{n-1}\left(\mathbb{C}^{n} \backslash\{0\}, \mathcal{O}_{\mathbb{C}^{n}}\right)$.

For $\delta \in \mathcal{D e r}_{\mathbb{C}}\left(\mathcal{O}_{\mathbb{C}^{n}}\right)$, we denote by $d_{\delta}$ the homomorphism

$$
d_{\delta}: \check{H}^{n-1}\left(V \backslash\{0\}, \mathcal{O}_{\mathbb{C}^{n}}\right) \rightarrow \check{H}^{n-1}\left(V \backslash\{0\}, \mathcal{O}_{\mathbb{C}^{n}}\right), \quad d_{\delta}([g])=[\delta \cdot g] .
$$

Proposition 3.1. Let $\mathbf{m}_{\mathbb{C}^{n}, 0}$ be the maximal ideal of $\mathcal{O}_{\mathbb{C}^{n}, 0}$ and let $\delta \in$ $\mathbf{m}_{\mathbb{C}^{n}, 0} \operatorname{Der}_{\mathbb{C}}\left(\mathcal{O}_{\mathbb{C}^{n}}\right)$,

$$
\delta=\left(x_{1}, \cdots, x_{n}\right)\left(\begin{array}{ccc}
a_{1,1} & \cdots & a_{1, n} \\
\vdots & \vdots & \vdots \\
a_{n, 1} & \cdots & a_{n, n}
\end{array}\right)\left(\begin{array}{c}
\partial / \partial x_{1} \\
\vdots \\
\partial / \partial x_{n}
\end{array}\right)+\delta_{\geq 1}
$$

with the $a_{i, j} \in \mathbb{C}$ and $\delta_{\geq 1} \in \mathbf{m}_{\mathbb{C}^{n}, 0}^{2} \mathcal{D e r}_{\mathbb{C}}\left(\mathcal{O}_{\mathbb{C}^{n}}\right)$. If $d_{\delta}$ is injective, then the eigenvalues of $A$ do not satisfy any relation with positive integer coefficients (in this case, we will say that $\delta$ satisfies condition (I)).

Proof. By a coordinate change we can make $A$ lower triangular. Its eigenvalues $a_{1}, \cdots, a_{n}$ are then the elements of the diagonal. The group $\check{H}^{n-1}\left(V \backslash\{0\}, \mathcal{O}_{\mathbb{C}^{n}}\right)$ is isomorphic to the space of Laurent series, convergent for all $\underline{x}=\left(x_{1}, \cdots, x_{n}\right)$ with $\underline{x} \neq 0$, whose non-zero coefficients are those with strictly negative indices in all variables, i.e.

$$
\sum_{i_{1}, \cdots, i_{n}<0} a_{i_{1}, \cdots, i_{n}} x_{1}^{i_{1}} \cdots x_{n}^{i_{n}}
$$

For $p \geq n$, we set

$$
\begin{aligned}
& G^{p}=\left\{\begin{array}{c}
\sum_{i_{n}<0} c_{\mathbf{i}} x_{1}^{i_{1}} \cdots x_{n}^{i_{n}} \\
i_{1}, \cdots, i_{n} \\
i_{1}+\cdots+i_{n}=-p
\end{array}\right\}, \\
& F^{p}=\left\{\begin{array}{c}
\sum_{i_{n}<0} \\
c_{\mathbf{i}} x_{1}^{i_{1}} \cdots x_{n}^{i_{n}} \\
i_{1}, \cdots, i_{n}< \\
i_{1}+\cdots+i_{n} \geq-p
\end{array}\right\} .
\end{aligned}
$$

Then $F^{p}=G^{p} \oplus G^{p-1} \oplus \cdots \oplus G^{n}$. Each $G^{p}$ is a finite-dimensional $\mathbb{C}$-vector space, whose dimension we denote by $r_{p}$, and $d_{\delta}$ restricts to morphisms of vector spaces

$$
d_{\delta \mid F^{p}}: F^{p} \rightarrow F^{p}
$$

and

$$
d_{\delta \mid G^{p}}: G^{p} \rightarrow F^{p}
$$


Let us denote by $d_{\delta, p}^{p}$ the component of this second restriction lying in $G^{p}$. Then $d_{\delta, p}^{p}$ depends only on the weight 0 part $\delta_{0}$ of $\delta$. We claim that with respect to a suitable ordered basis of $G^{p}$, its matrix $\left[d_{\delta, p}^{p}\right]$ is lower triangular.

As basis for $G^{p}$ we take the monomials

$$
\frac{1}{x_{1}^{i_{1}} \cdots x_{n}^{i_{n}}}
$$

with $i_{1}+\cdots+i_{n}=p$.

We have

$$
d_{\delta}\left(x_{1}^{-i_{1}} \cdots x_{n}^{-i_{n}}\right)=-\sum_{j, k} i_{k} a_{j, k} x_{1}^{-i_{1}} \cdots x_{k}^{-\left(i_{k}-1\right)} \cdots x_{j}^{-\left(i_{j}+1\right)} \cdots x_{n}^{-i_{n}} .
$$

Thus, if we give our basis of $G^{p}$ the lexicographic order corresponding to the order of the coordinates $x_{1}, \cdots, x_{n}$, then since $a_{j, k}=0$ if $j<k$ (recall that we have chosen our coordinates so that $A$ is lower triangular), the matrix $\left[d_{\delta, p}^{p}\right]$ is lower triangular.

Let $q \leq p$. Then $d_{\delta}\left(G^{q}\right) \subset G^{q}+G^{q-1}+\cdots+G^{n}$. Thus, it follows from the above that if we give $F^{p}$ the ordered basis consisting of the ordered bases for each $G^{q}, n \leq q \leq p$ that we have chosen, and order these by descending value of $q$, then the matrix of $d_{\delta \mid F^{p}}$ is also lower triangular.

What are its diagonal elements? In the right-hand side of equation (1), the coefficient of $x_{1}^{-i_{1}} \cdots x_{n}^{-i_{n}}$ is equal to

$$
i_{1} a_{1,1}+\cdots+i_{n} a_{n, n}
$$

this is the diagonal element in the matrix of $d_{\delta \mid F^{p}}$ in the row and column corresponding to the basis element $x_{1}^{-i_{1}} \cdots x_{n}^{-i_{n}}$. Note that the diagonal elements of $A$ are its eigenvalues; thus, the diagonal elements in the matrix of $d_{\delta \mid F^{p}}$ with respect to the chosen basis are all linear combinations $i_{1} \lambda_{1}+\cdots+i_{n} \lambda_{n}$ of the eigenvalues $\lambda_{1}, \cdots, \lambda_{n}$ of $A$, with the $i_{j}$ positive integers and $i_{1}+\cdots+i_{n} \leq p$. As this matrix is lower triangular, $d_{\delta} \mid F^{p}$ is injective only if the product of these diagonal elements is non-zero.

Remark 3.2. We have used in the proof of this lemma the fact that if $d_{\delta}$ is injective then so is its restriction to each $F^{p}$. We do not know if the opposite implication holds. It seems likely that an argument involving faithful flatness would prove it. However, we do not need it in what follows.

Let $D$ be a plane curve. We suppose as above that 0 is the singular point of $D$. In this case the upper non-zero row in the $E_{2}$ page of the spectral sequence ${ }^{\prime} \tilde{E}$ begins

$$
d_{1}: \check{H}^{1}\left(\mathbb{C}^{2} \backslash\{0\}, \mathcal{O}_{\mathbb{C}^{2}}\right) \rightarrow \oplus_{1}^{2} \check{H}^{1}\left(\mathbb{C}^{2} \backslash\{0\}, \mathcal{O}_{\mathbb{C}^{2}}\right)
$$


Theorem 3.3. Let $D$ be a plane curve, singular at 0 . If $d_{1}$ is injective, then there is a local equation $h$ for $D$ around 0 , and a germ of vector field $\chi$ at 0 such that $\chi \cdot h=h$.

Proof. Any reduced plane curve whose equation has non-zero quadratic part is quasihomogeneous, by the classification of singularities of functions of two variables: such a curve is equivalent to $A_{k}, x^{2}+y^{k+1}=0$, for some $k$. For a quasihomogeneous curve, the conclusion of the theorem of course holds. Thus, we may assume that the equation $h$ of $D$ lies in $\mathbf{m}_{\mathbb{C}^{2}, 0}^{3}$. As the determinant of the coefficients of a free basis of $\mathcal{D} \operatorname{er}(\log D)$ is a local defining equation for $D([11])$, we may therefore choose a free basis $\delta, \gamma$ for $\mathcal{D} \operatorname{er}(\log D)$ such that $\gamma$ has zero linear part. In fact the supposition that $d_{1}$ is injective implies that at least one member of the basis has non-zero linear part, as otherwise $d_{1}([1 / x y])=([\delta \cdot 1 / x y],[\gamma \cdot 1 / x y])=0$.

We may thus take

$$
\delta=\delta_{0}+\delta_{1}+\delta_{2}+\cdots=\sum_{k \geq 0} \sum_{i+j=k+1}\left(\alpha_{i j} x^{i} y^{j} \frac{\partial}{\partial x}+\beta_{i j} x^{i} y^{j} \frac{\partial}{\partial y}\right)
$$

where $\delta_{0}=\underline{x} A \underline{\partial}_{x}{ }^{t}$, with $A \neq 0$ and in Jordan normal form, i.e.

$$
A=\left(\begin{array}{cc}
\lambda_{1} & 0 \\
0 & \lambda_{2}
\end{array}\right) \text { or } A=\left(\begin{array}{cc}
\lambda_{1} & 0 \\
1 & \lambda_{1}
\end{array}\right) .
$$

Let $h$ be the reduced equation of $D$ :

$$
h=h_{n}+h_{n+1}+h_{n+2}+\cdots=\sum_{k \geq n} h_{k}=\sum_{k \geq n} \sum_{i+j=k} a_{i j} x^{i} y^{j},
$$

where the polynomials $h_{i}$ are homogeneous of degree $i$.

Let us now suppose that $\delta$ is not an Euler vector field for $h$, we will see that (up to multiplication by a non-zero constant) the only possibility for $h$ and $\delta$ is

$$
h_{1}=\cdots=h_{n-1}=0, h_{n}=x^{a} y^{b} \quad \text { and } \quad \delta_{0}=q x \frac{\partial}{\partial x}-p y \frac{\partial}{\partial y} .
$$

First case: $h_{n}=\sum_{i+j=n} a_{i j} x^{i} y^{j}$ and $\delta_{0}=\lambda_{1} x \frac{\partial}{\partial x}+\lambda_{2} y \frac{\partial}{\partial y}$. Then

$$
0=\delta_{0}\left(h_{n}\right)=\sum_{i+j=n}\left(i \lambda_{1}+j \lambda_{2}\right) a_{i j} x^{i} y^{j} .
$$

So, $a_{i j}=0$ if $i \lambda_{1}+j \lambda_{2} \neq 0$; thus, since by assumption $h_{n} \neq 0$, we have $q \lambda_{1}=-p \lambda_{2}$ and $p+q=n(p, q \in \mathbb{N})$. In this case,

$$
h_{n}=x^{p} y^{q}, \quad \delta_{0}=q x \frac{\partial}{\partial x}-p y \frac{\partial}{\partial y} .
$$

Second case: $h_{n}=\sum_{i+j=n} a_{i j} x^{i} y^{j}$ and $\delta_{0}=\left(\lambda_{1} x+y\right) \frac{\partial}{\partial x}+\lambda_{1} y \frac{\partial}{\partial y}$. Then

$$
0=\delta_{0}\left(h_{n}\right)=n \lambda_{1} a_{n 0} x^{n}+\sum_{i+j=n, j \geq 1}\left(n \lambda_{1} a_{i j}+i a_{i+1, j-1}\right) x^{i} y^{j} .
$$


So, if $\lambda_{1} \neq 0$, then we must have $a_{n 0}=0$, then $a_{n-1,1}=0, \cdots, a_{1, n-1}=0, a_{0 n}=0$, so that $h_{n}=0$. This is absurd, by hypothesis.

If $\lambda_{1}=0$, then $d_{1}$ is not injective, because

$$
d_{1}([1 / x y])=\left(d_{\delta}([1 / x y]), d_{\gamma}([1 / x y])\right)=(0,0) .
$$

Then, we have

$$
h=x^{p} y^{q}+h_{n+1}+h_{n+2}+\cdots, \quad \delta_{0}=q x \frac{\partial}{\partial x}-p y \frac{\partial}{\partial y} .
$$

We will prove that, in this case, after a coordinate change $h$ can be reduced to $h=x^{p} y^{q}$ with $p+q=n \geq 3$. This contradicts our supposition that $h$ is reduced. Then our initial supposition about $\delta$ is false, and $\delta$ is an Euler vector field for $h$.

Inductively, for all $k \geq 0$, we construct coordinates $\left(x_{(k)}, y_{(k)}\right)$ and functions $h^{(k)}$ such that

$$
h(x, y)=h^{(k)}\left(x_{(k)}, y_{(k)}\right)=x_{(k)}^{p} y_{(k)}^{q}+\sum_{s \geq n+k} h_{s}^{(k)}\left(x_{(k)}, y_{(k)}\right) \equiv x_{(k)}^{p} y_{(k)}^{q}\left(\mathbf{m}_{\mathbb{C}^{2}, 0}^{n+k}\right),
$$

where $h_{i}^{(k)}$ is homogeneous of degree $i$. Then, by Artin approximation [1, Theorem $1.2]$, there exist coordinates $z_{1}, z_{2}$ solving the equation

$$
h(x, y)-z_{1}^{p} z_{2}^{q}=0 .
$$

Let us construct the $x_{(k)}, y_{(k)}, h^{(k)}$. We suppose that we have $x_{(k)}, y_{(k)}$ and $h^{(k)} \in$ $\mathbb{C}\left\{x_{(k)}, y_{(k)}\right\}$, such that

$$
\begin{gathered}
h(x, y)=h^{(k)}\left(x_{(k)}, y_{(k)}\right)=x_{(k)}^{p} y_{(k)}^{q}+\sum_{s \geq n+k} h_{s}^{(k)}, \\
\delta_{0}^{(k)}=q x_{(k)} \frac{\partial}{\partial x_{(k)}}-p y_{(k)} \frac{\partial}{\partial y_{(k)}} .
\end{gathered}
$$

We define $x_{(k+1)}, y_{(k+1)}$ and $h^{(k+1)} \in \mathbb{C}\left\{x_{(k+1)}, y_{(k+1)}\right\}$, such that

$$
\begin{gathered}
h(x, y)=h^{(k+1)}\left(x_{(k+1)}, y_{(k+1)}\right)=x_{(k+1)}^{p} y_{(k+1)}^{q}+\sum_{s \geq n+k+1} h_{s}^{(k+1)}, \\
\delta_{0}^{(k+1)}=q x_{(k+1)} \frac{\partial}{\partial x_{(k+1)}}-p y_{(k+1)} \frac{\partial}{\partial y_{(k+1)}} .
\end{gathered}
$$

Let $h_{n+k}^{(k)}=\sum_{i+j=n+k} a_{i, j}^{(k)} x_{(k)}^{i} y_{(k)}^{j}$, then

$$
\delta_{0}^{(k)}\left(h_{n+k}\right)=\sum_{i+j=n+k}(i q-j p) a_{i, j}^{(k)} x_{(k)}^{i} y_{(k)}^{j}
$$

As the part of $h^{(k)}$ of degree less than $n+k$ is $x_{(k)}^{p} y_{(k)}^{q}$, it follows that the part of degree $n+k$ of $\delta^{(k)}\left(h^{(k)}\right) \in \mathbf{m}_{\mathbb{C}^{2}, 0} h^{(k)}$ belongs to $\left(x_{(k)}^{p} y_{(k)}^{q}\right)$ :

$$
\left[\delta^{(k)}\left(h^{(k)}\right)\right]_{n+k}=\delta_{0}^{(k)}\left(h_{n+k}^{(k)}\right)+\delta_{k}^{(k)}\left(x_{(k)}^{p} y_{(k)}^{q}\right) \in\left(x_{(k)}^{p} y_{(k)}^{q}\right),
$$


but

$$
\delta_{k}^{(k)}\left(x_{(k)}^{p} y_{(k)}^{q}\right) \in\left(x_{(k)}^{p-1} y_{(k)}^{q}, x_{(k)}^{p} y_{(k)}^{q-1}\right)
$$

then

$$
\delta_{0}^{(k)}\left(h_{n+k}^{(k)}\right) \in\left(x_{(k)}^{p-1} y_{(k)}^{q}, x_{(k)}^{p} y_{(k)}^{q-1}\right)
$$

SO

$$
(i q-j p) a_{i, j}^{(k)}=0(i+j=n+k) \text { if } i<p-1 \text { or } j<q-1,
$$

but if $i q-j p=0$, then $(i, j)=\frac{n+k}{n}(p, q)$, and $i>p, j>q$. So $h_{n+k}^{(k)} \in$ $\left(x_{(k)}^{p-1} y_{(k)}^{q}, x_{(k)}^{p} y_{(k)}^{q-1}\right):$

$$
h_{n+k}^{(k)}=x_{(k)}^{p-1} y_{(k)}^{q} f_{k+1}\left(x_{(k)}, y_{(k)}\right)+x_{(k)}^{p} y_{(k)}^{q-1} g_{k+1}\left(x_{(k)}, y_{(k)}\right) .
$$

Let

$$
x_{(k+1)}=x_{(k)}+\frac{1}{p} f_{k+1}\left(x_{(k)}, y_{(k)}\right) \quad y_{(k+1)}=y_{(k)}+\frac{1}{q} g_{k+1}\left(x_{(k)}, y_{(k)}\right) .
$$

We have

$$
h(x, y)=x_{(k+1)}^{p} y_{(k+1)}^{q}+\sum_{r \geq k+1} \sum_{i+j=n+r} a_{i, j}^{(k+1)} x_{(k+1)}^{i} y_{(k+1)}^{j} .
$$

We define $h^{(k+1)}$ by the equation $h(x, y)=h^{(k+1)}\left(x_{(k+1)}, y_{(k+1)}\right)$, where

$$
h^{(k+1)}=x_{(k+1)}^{p} y_{(k+1)}^{q}+\sum_{s \geq n+k+1} h_{s}^{(k+1)},
$$

with $h_{s}^{(k+1)}=\sum_{i+j=s} a_{i, j}^{(k+1)} x_{(k+1)}^{i} y_{(k+1)}^{j}$ homegeneous polynomials of degree $s \geq$ $n+k+1$. Moreover, as

$$
x_{(k+1)}=x_{(k)} ; \quad y_{(k+1)}=y_{(k)}\left(\bmod \mathbf{m}_{\mathbb{C}^{2}, 0}^{2}\right),
$$

we have $\delta=\sum_{q \geq 0} \delta_{q}^{(k+1)}$, where each $\delta_{q}^{(k+1)}$ is homogeneous of degree $q$, and

$$
\delta_{0}^{(k+1)}=q x_{(k+1)} \frac{\partial}{\partial x_{(k+1)}}-p y_{(k+1)} \frac{\partial}{\partial y_{(k+1)}} .
$$

Proposition 3.4. Let $D$ a plane curve, singular at 0 . If there exists $\delta \in \mathcal{D} \operatorname{er}(\log D)$ satisfying condition (I), then there exists a unit $\alpha$ such that $\alpha \delta \cdot h=h$, and so $D$ is Euler homogeneous.

Proof. The proof is similar to the proof of Theorem 3.3. There, we consider the case where $h_{n}=x^{p} y^{q}$ and $\delta_{0}=q x \partial / \partial x-p y \partial / \partial y$, with $p, q \in \mathbb{N}$. Condition (I) forces one of $p$ and $q$ to be 0 . The proof now proceeds as before, with this additional hypothesis. 
Theorem 3.5. Let $(D, 0) \subset\left(\mathbb{C}^{2}, 0\right)$ be a plane curve. The following conditions are equivalent:

a) There exists $\delta \in \mathcal{D} \operatorname{er}(\log D)_{0}$ such that $d_{\delta}$ is injective.

b) There exists $\delta \in \mathcal{D} \operatorname{er}(\log D)_{0}$ satisfying condition (I).

c) $d_{1}$ is injective.

d) $(D, 0)$ is Euler homogeneous.

e) $(D, 0)$ is quasi-homogeneous.

f) The logarithmic comparison theorem holds for $(D, 0)$ on a neighbourhood of 0 .

Proof. By Theorem 3.3, if $d_{1}$ is injective, then $(D, 0)$ is Euler homogeneous. By Saito's theorem [10] (for a function $h$ with isolated singularity, $h \in J_{h}$ is equivalent to the quasihomogeneity of $h$ ) to be Euler homogeneous or quasi-homogeneous is the same. Theorem 1.1 proves that if $(D, 0)$ is quasi-homogeneous, the logarithmic comparison theorem holds for $(D, 0)$ on a neighborough of 0 . From the results of section 2 we can easily deduce that logarithmic comparison theorem implies the injectivity of $d_{1}$. Then, the last four conditions are equivalent. If $\chi=w_{1} \frac{\partial}{\partial x}+w_{2} \frac{\partial}{\partial y}$ is the Euler vector field then $d_{\chi}$ is injective. Proposition 3.1 shows that if $d_{\delta}$ is injective, then $\delta$ satisfies (I) and, finally, by proposition $3.4, \delta \in \mathcal{D} \operatorname{er}(\log D)$ implies that $D$ is Euler homogeneous.

\section{Example}

In this section we give an example of a free divisor $D \subset \mathbb{C}^{3}$ which is Euler homogeneous but not locally quasi-homogeneous, and for which the logarithmic comparison theorem does hold. This example is studied in [4], where the perversity of $\Omega^{\bullet}(\log D)$ is proved. We remark that $D$ is the total space of an equisingular one-parameter deformation of a plane curve singularity. In [7], Damon shows that under mild additional hypotheses, all surfaces obtained in this way are free divisors.

$D$ is defined by the equation

$$
h(x, y, z)=x y(x+y)((z-\lambda) x+y)=h_{1} h_{2} h_{3} h_{4}, \quad \lambda \in \mathbb{C} \backslash\{0,1\} .
$$

$\mathcal{D}$ er $(\log D)$ has free basis $\left\{\delta_{1}, \delta_{2}, \delta_{3}\right\}$

$$
\begin{aligned}
& \delta_{1}=x \frac{\partial}{\partial x}+y \frac{\partial}{\partial y}+((z-\lambda) x+y) \frac{\partial}{\partial z} \\
& \delta_{2}= \\
& \delta_{3}=x^{2} \frac{\partial}{\partial x}-y^{2} \frac{\partial}{\partial y}-(z-\lambda)(x+y) \frac{\partial}{\partial z} .
\end{aligned}
$$

Note that $\delta_{1} \cdot h=4 h$, so that $h$ is Euler homogeneous. Note also that it is easy to check that each of these vector fields is logarithmic, and that the determinant of their coefficients is a reduced equation for $D$. From this it follows by a theorem 
of K. Saito ([11]) that they really do form a basis for $\mathcal{D} \operatorname{er}(\log D)$; as no linear combination of them has non-singular linear part, it follows that $D$ cannot be quasihomogeneous.

This example of free divisor is interesting also as it provides a counterexample to the "logarithmic Sard's theorem": every point of $\mathbb{C}=z$-axis is a logarithmic critical value with respect to the projection $(x, y, z) \mapsto z$.

The basis of $\Omega^{1}(\log D)$ dual to $\left\{\delta_{1}, \delta_{2}, \delta_{3}\right\}$ is

$$
\begin{aligned}
\omega_{1} & =\frac{y^{2} d x+x^{2} d y}{x y(x+y)} \\
\omega_{2} & =\frac{y(z-\lambda) d x-x(z-\lambda) d y+x y d z}{x y(x(z-\lambda)+y)} \\
\omega_{3} & =\frac{y d x-x d y}{x y(x+y)} .
\end{aligned}
$$

We have to calculate homology groups of the stalk at 0 of the logarithmic de Rham complex

$$
0 \rightarrow \Omega^{0}(\log D) \stackrel{d_{0}}{\rightarrow} \Omega^{1}(\log D) \stackrel{d_{1}}{\rightarrow} \Omega^{2}(\log D) \stackrel{d_{2}}{\rightarrow} \Omega^{3}(\log D) \stackrel{d_{3}}{\rightarrow} 0 .
$$

Although $D$ is not weighted homogeneous in the strict sense, it is homogeneous if we assign weights $1,1,0$ to the variables $x, y, z$. The Lie derivative with respect to the vector field $\delta_{1}$,

$$
L_{\delta_{1}}(\omega)=\iota_{\delta_{1}}(d \omega)+d\left(\iota_{\delta_{1}}(\omega)\right),
$$

then defines a contracting homotopy from $\Omega^{\bullet}(\log D)$ to its weight-zero part $\Omega_{0}^{\bullet}(\log D)$. For if $\omega \in \Omega^{k}(\log D)$ is a sum of homogenenous parts $\omega_{i}$, and if $d \omega=0$, then $d \omega_{i}=0$ for all $i$. Since $L_{\delta_{1}}\left(\omega_{i}\right)=i \omega_{i}$, each $\omega_{i}$, for $i \neq 0$, is then exact, and $\omega$ is cohomologous to $\omega-\iota_{\delta_{1}}\left(\sum_{i \neq 0}(1 / i) \omega_{i}\right)$.

Thus we consider only the weight 0 subcomplex

$$
0 \rightarrow \Omega_{0}^{0}(\log D) \stackrel{d_{0}^{0}}{\rightarrow} \Omega_{0}^{1}(\log D) \stackrel{d_{1}^{0}}{\rightarrow} \Omega_{0}^{2}(\log D) \stackrel{d_{2}^{0}}{\rightarrow} \Omega_{0}^{3}(\log D) \stackrel{d_{3}^{0}}{\rightarrow} 0 .
$$

- We have $\Omega_{0}^{0}(\log D)=\mathbb{C}\{z\}$, and $d_{0}\left(z^{k}\right)=k z^{k-1}\left[((z-\lambda) x+y) \omega_{2}-\right.$ $\left.(z-\lambda)(x+y) \omega_{3}\right](k \geq 0)$, so

$$
\operatorname{Im}\left(d_{0}^{0}\right)=\mathbb{C}\{z\} d z=\mathbb{C}\{z\}\left\langle((z-\lambda) x+y) \omega_{2}-(z-\lambda)(x+y) \omega_{3}\right\rangle .
$$

- $\Omega_{0}^{1}(\log D)=\mathbb{C}\{z\}\left\langle\omega_{1}, x \omega_{2}, y \omega_{2}, x \omega_{3}, y \omega_{3}\right\rangle$, and we find

$$
\begin{aligned}
d_{1}\left(\omega_{1}\right) & =d_{1}\left(x \omega_{2}\right)=d_{1}\left(x \omega_{3}\right)=d_{1}\left(y \omega_{3}\right)=0 \\
d_{1}\left(z^{k} \omega_{1}\right) & =k z^{k-1}\left((x(\lambda-z)-y) \omega_{1} \wedge \omega_{2}+(z-\lambda)(x+y) \omega_{1} \wedge \omega_{3}\right) \\
d_{1}\left(y \omega_{2}\right) & =\left(x y+y^{2}\right) \omega_{2} \wedge \omega_{3} \\
d_{1}\left(z^{k} x \omega_{2}\right) & =k z^{k-1}\left((z-\lambda)(x+y) x \omega_{2} \wedge \omega_{3}\right) \\
d_{1}\left(z^{k} y \omega_{2}\right) & =\left((k+1) z^{k}-k \lambda z^{k-1}\right)(x+y) y \omega_{2} \wedge \omega_{3} \\
d_{1}\left(z^{k} x \omega_{3}\right) & =k z^{k-1} x(x(z-\lambda)+y) \omega_{2} \wedge \omega_{3} \\
d_{1}\left(z^{k} y \omega_{3}\right) & =k z^{k-1} y(x(z-\lambda)+y) \omega_{2} \wedge \omega_{3} .
\end{aligned}
$$


It follows that $\operatorname{Ker}\left(d_{1}^{0}\right)=\mathbb{C}\left\langle\omega_{1}, x \omega_{2}, x \omega_{3}, y \omega_{3}\right\rangle \oplus \operatorname{Im}\left(d_{0}^{0}\right)$, so

$$
h^{1}\left(\Omega^{\bullet}(\log D)_{0}\right)=\mathbb{C}\left\langle\omega_{1}, x \omega_{2}, x \omega_{3}, y \omega_{3}\right\rangle
$$

is 4-dimensional. Also we have

$$
\begin{gathered}
\left.\operatorname{Im}\left(d_{1}^{0}\right)=\mathbb{C}\{z\}\left\langle((\lambda-z) x-y) \omega_{1} \wedge \omega_{2}+(z-\lambda)(x+y) \omega_{1} \wedge \omega_{3}\right)\right\rangle \oplus \\
\mathbb{C}\{z\}\left\langle x^{2}, x y, y^{2}\right\rangle \omega_{2} \wedge \omega_{3} .
\end{gathered}
$$

- $\Omega_{0}^{2}(\log D)$ is generated over $\mathbb{C}\{z\}$ by

$x \omega_{1} \wedge \omega_{2}, y \omega_{1} \wedge \omega_{2}, x \omega_{3} \wedge \omega_{1}, y \omega_{3} \wedge \omega_{1}, x^{2} \omega_{2} \wedge \omega_{3}, x y \omega_{2} \wedge \omega_{3}, y^{2} \omega_{2} \wedge \omega_{3}$.

We find

$$
\begin{aligned}
d_{2}\left(x \omega_{1} \wedge \omega_{2}\right) & =d_{2}\left(x \omega_{1} \wedge \omega_{3}\right)=d_{2}\left(y \omega_{1} \wedge \omega_{3}\right)=0 \\
d_{2}\left(z^{k} x^{2} \omega_{2} \wedge \omega_{3}\right) & =d_{2}\left(z^{k} x y \omega_{2} \wedge \omega_{3}\right)=d_{2}\left(z^{k} y^{2} \omega_{2} \wedge \omega_{3}\right)=0 . \\
d_{2}\left(z^{k} x \omega_{1} \wedge \omega_{2}\right) & =k z^{k-1}(\lambda-z)(x+y) x \omega_{1} \wedge \omega_{2} \wedge \omega_{3} \\
d_{2}\left(y \omega_{1} \wedge \omega_{2}\right) & =\left(x y+y^{2}\right) \omega_{1} \wedge \omega_{2} \wedge \omega_{3} \\
d_{2}\left(z^{k} y \omega_{1} \wedge \omega_{2}\right) & \left.=z^{k-1}(x+y)(k y(\lambda-z)-z y) \omega_{1} \wedge \omega_{2} \wedge \omega_{3}\right) \\
d_{2}\left(z^{k} x \omega_{1} \wedge \omega_{3}\right) & =-k z^{k-1} x((z-\lambda) x+y) \omega_{1} \wedge \omega_{2} \wedge \omega_{3} \\
d_{2}\left(z^{k} y \omega_{1} \wedge \omega_{3}\right) & =-k z^{k-1} y((z-\lambda) x+y) \omega_{1} \wedge \omega_{2} \wedge \omega_{3} .
\end{aligned}
$$

We deduce that $\operatorname{Ker}\left(d_{2}^{0}\right)=\mathbb{C}\left\langle x \omega_{1} \wedge \omega_{2}, x \omega_{1} \wedge \omega_{3}, y \omega_{1} \wedge \omega_{3}\right\rangle \oplus \operatorname{Im}\left(d_{1}^{0}\right)$, and thus that

$$
h^{2}\left(\Omega^{\bullet}(\log D)_{0}\right)=\mathbb{C}\left\langle x \omega_{1} \wedge \omega_{2}, x \omega_{1} \wedge \omega_{3}, y \omega_{1} \wedge \omega_{3}\right\rangle
$$

is 3-dimensional.

- Finally,

$$
\operatorname{Im}\left(d_{2}^{0}\right)=\mathbb{C}\{z\}\left\langle x^{2}, x y, y^{2}\right\rangle \omega_{1} \wedge \omega_{2} \wedge \omega_{3}=\Omega_{0}^{3}(\log D),
$$

and, consequently,

$$
h^{3}\left(\Omega^{\bullet}(\log D)_{0}\right)=0 .
$$

Now consider the intersection $D_{0}=D \cap\{z=0\}$, which has equation

$$
h^{0}=h_{1}^{0} h_{2}^{0} h_{3}^{0} h_{4}^{0}=x y(x+y)(-\lambda x+y) .
$$

It is a line arrangement, and the cohomology of its complement is therefore given by the Brieskorn complex, the exterior algebra generated over $\mathbb{C}$ by the forms $d h_{i}^{0} / h_{i}^{0}$, with trivial differential ([2]). This is of course a subcomplex of $\Omega^{\bullet}\left(\log D_{0}\right)$. Let $V \subset \mathbb{C}^{3}$ be a neighbourhood of 0 . Restriction from $\mathbb{C}^{3}$ to $\mathbb{C}^{2}=\{z=0\}$ gives rise to a commutative diagram

$$
\begin{array}{ccc}
\wedge^{p} \sum_{1 \leq i \leq 4} \mathbb{C}\left\langle\frac{d h_{i}}{h_{i}}\right\rangle \stackrel{a}{\longrightarrow} h^{p}\left(\Omega^{\bullet}(\log D)(V)\right) & \stackrel{b}{\longrightarrow} H^{p}(V \backslash D ; \mathbb{C}) \\
\downarrow & \downarrow & \downarrow \cong \\
\wedge^{p} \sum_{1 \leq i \leq 4} \mathbb{C}\left\langle\frac{d h_{i}^{0}}{h_{i}^{0}}\right\rangle \stackrel{\cong}{\longrightarrow} h^{p}\left(\Omega^{\bullet}\left(\log D_{0}\right)\left(V_{0}\right)\right) \stackrel{\cong}{\longrightarrow} H^{p}\left(V_{0} \backslash D_{0} ; \mathbb{C}\right) .
\end{array}
$$


in which the left-hand horizontal morphisms are induced by the inclusion of the Brieskorn complex in the logarithmic complex, and the right-hand horizontal morphisms are de Rham maps. The lower horizontal morphisms are isomorphisms by the theorem of Brieskorn and by 1.1. The right-hand vertical morphism is an isomorphism because $D$ is a topologically trivial deformation of $D_{0}$, so inclusion induces an isomorphism of the homology groups of the complements. The left-hand vertical morphism is evidently surjective, and thus the de Rham map $h^{p}\left(\Omega^{\bullet}(\log D)(V)\right) \rightarrow H^{p}(V \backslash D ; \mathbb{C})$ is surjective. As $h^{p}\left(\Omega^{\bullet}(\log D)_{0}\right)=$ $\lim _{U \ni 0} h^{p}\left(\Omega^{\bullet}(\log D)(V)\right)$ and $\lim _{U \ni 0} H^{p}(V \backslash D ; \mathbb{C})=H^{p}\left(\mathbb{C}^{3} \backslash D ; \mathbb{C}\right)$, then the de Rham map $h^{p}\left(\Omega^{\bullet}(\log D)\right) \rightarrow H^{p}\left(\mathbb{C}^{3} \backslash D ; \mathbb{C}\right)$ is surjective. To see that it is an isomorphism we compare dimensions. A calculation (for example, using the Brieskorn complex) gives

$$
\begin{aligned}
& \operatorname{dim}_{\mathbb{C}} H^{1}\left(\mathbb{C}^{2} \backslash D_{0} ; \mathbb{C}\right)=4 \\
& \operatorname{dim}_{\mathbb{C}} H^{2}\left(\mathbb{C}^{2} \backslash D_{0} ; \mathbb{C}\right)=3 \\
& \operatorname{dim}_{\mathbb{C}} H^{3}\left(\mathbb{C}^{2} \backslash D_{0} ; \mathbb{C}\right)=0 .
\end{aligned}
$$

As these are the same as the dimension of $h^{p}\left(\Omega^{\bullet}(\log D)_{0}\right)$, this completes the proof that the logarithmic comparison theorem holds for $D$.

Remark 4.1. The calculations whose results we summarise here are not so simple as might be supposed. We have presented each image $d_{i}^{0}\left(\Omega_{0}^{i}(\log D)\right)$ as a module over $\mathbb{C}\{z\}$ with algebraic generators, obscuring the fact that because $D$ is not quasihomogeneous, the anti-derivatives of an algebraic exact logarithmic form are in general transcendental. For example,

$$
\begin{aligned}
z^{k}\left(x^{2}+x y\right) \omega_{1} \wedge \omega_{2} \wedge \omega_{3} & =d\left(\sum_{s=1}^{\infty}\left(z^{k+s} / \lambda^{s}(k+s)\right) x \omega_{1} \wedge \omega_{2}\right) \\
& =d\left(-\left(\log \left(1-\frac{z}{\lambda}\right)+\sum_{s=1}^{k}\left(z^{s} / \lambda^{s} s\right)\right) \lambda^{k} x \omega_{1} \omega_{2}\right)
\end{aligned}
$$

and

$$
\begin{aligned}
z^{k} x y \omega_{1} \wedge \omega_{2} \wedge \omega_{3}=d & \left(\sum_{s=1}^{\infty}\left(z^{k+s} /(\lambda+1)^{s}(k+s)\right) x\left(\omega_{1} \wedge \omega_{2}+\omega_{1} \wedge \omega_{3}\right)\right) \\
=d & -\left((\lambda+1)^{k} \log (1-(z /(\lambda+1)))\right. \\
& \left.\left.+\sum_{s=1}^{k}\left(z^{s}(\lambda+1)^{k-s} s\right)\right) x\left(\omega_{1} \wedge \omega_{2}+\omega_{1} \wedge \omega_{3}\right)\right)
\end{aligned}
$$




\section{References}

[1] M. Artin, On the solutions of analytic equations, Invent. Math. 5 (1968), 277-291.

[2] E. Brieskorn, Sur le groupe de tresses (d'apres V. I. Arnol'd), Sem. Bourbaki 1971/72, Lecture Notes in Math. 317, Springer Verlag, Berlin, 1973, 21-44.

[3] J. L. Brylinski, A. S. Dubson and M. Kashiwara, Formule de l'indice pour modules holonomes et obstruction d'Euler locale, C. R. Acad. Sci. Paris Sér. I Math., 293 (1981), $573-576$.

[4] F. J. Calderón Moreno, Logarithmic Differential Operators and Logarithmic De Rham Complexes Relative to a Free Divisor, Ann. Sci. École Norm. Sup. (4) 32 (1999), no. 5, $701-714$.

[5] F. J. Castro Jiménez, D. Mond and L. Narváez Macarro, Cohomology of the complement of a free divisor, Transactions of the A.M.S. 348 (1996), 3037-3049.

[6] F. J. Castro Jiménez, D. Mond and L. Narváez Macarro, Unpublished, 1997.

[7] J. N. Damon. On the freeness of equisingular deformations of plane curve singularities, Topology and Applications, to appear.

[8] A. Grothendieck, On the de Rham cohomology of algebraic varieties, Publ. Math. de l'I.H.E.S. 29 (1966), 95-103.

[9] H. J. Reiffen, Das Lemma von Poincaré für holomorphe Differentialformen auf komplexen Raumen, Math. Z. 101 (1967), 269-284.

[10] K. Saito, Quasihomogene isolierte Singularitäten von Hyperflächen, Invent. Math. 14 (1971), 123-141.

[11] K. Saito, Theory of logarithmic differential forms and logarithmic vector fields, J. Fac. Sci. Univ. Tokyo 27 (1980), 265-291.

Francisco J. Calderón Moreno

Universidad de Sevilla

Facultad de Matemáticas

Departamento de Álgebra

Apartado postal 1160

41080 Sevilla

Spain

e-mail: calderon@algebra.us.es frcalder@us.es

Francisco J. Castro Jiménez

Universidad de Sevilla

Facultad de Matemáticas

Departamento de Álgebra

Apartado postal 1160

41080 Sevilla

Spain

e-mail: castro@algebra.us.es

(Received: February 25, 1999)
Luis Narváez Macarro

Universidad de Sevilla

Facultad de Matemáticas

Departamento de Álgebra

Apartado postal 1160

41080 Sevilla

Spain

e-mail: narvaez@algebra.us.es

David Mond

University of Warwick

Mathematics Institute

Coventry CV4 7AL

England

e-mail: mond@maths.warwick.ac.uk 